\title{
REDUÇÃO DO TEMPO DE HOSPITALIZAÇÃO APÓS CIRURGIA TORÁCICA DE GRANDE PORTE: IDENTIFICAÇÃO DE FATORES DETERMINANTES
}

\author{
EARLY DISCHARGE FOLLOWING MAJOR THORACIC SURGERY: \\ IDENTIFICATION OF RELATED FACTORS
}

\author{
Nuno Fevereiro Ferreira de Lima ${ }^{1}$ \\ André Luís de Aquino Carvalho, TCBC-DF ${ }^{2}$
}

\begin{abstract}
RESUMO: Objetivo: Os custos da internação hospitalar têm relação direta com o tempo de permanência do paciente operado. Por outro lado, um menor tempo de internação permite aumentar a produtividade nos hospitais públicos com demanda reprimida. O objetivo deste estudo é identificar fatores determinantes da redução do tempo de internação pós-cirurgia torácica de grande porte. Método: Estudo retrospectivo por análise de prontuários, realizado em duas fases. Na fase I levantou-se uma série consecutiva de 169 pacientes divididos em grupo I ( $\mathrm{n}=81$ ) - operados no período de junho de 1990 a dezembro de 1995, e grupo II ( $\mathrm{n}=88)$ operados de janeiro de 1996 a maio de 2000, para verificação do tempo de internação e fatores relacionados. Na fase II levantou-se uma série consecutiva de 20 pacientes (grupo III) retroagindo a partir de março de 2002, para análise e comparação com uma pequena enquête enviada pela internet para 21 cirurgiões torácicos. Resultados: A quase totalidade dos pacientes prescindiu de UTI no pós-operatório imediato. O tempo de internação médio caiu de 7,6 dias (mediana 7) no grupo I para 5,1 dias (mediana 4) no grupo II (p<0,001). A utilização mais freqüente da analgesia epidural e o uso de vias de acesso menos traumáticas no grupo II alcançou significância estatística ( $\mathrm{p}<0,001)$. No grupo III o tempo de internação médio foi de 4,2 dias (mediana 4), com uso mais efetivo da analgesia epidural (75\%) e emprego da toracotomia vertical (90\%). Oito cirurgiões torácicos responderam à enquête: o tempo médio de internação informado variou de cinco a nove dias, todos os pacientes foram enviados para a UTI ou similar, e apenas dois cirurgiões usam de rotina toracotomia com preservação muscular. Conclusões: Este estudo confirma que o controle da dor e o menor trauma da via de acesso são fatores importantes para a mais rápida recuperação funcional dos pacientes. Sugere que uso da UTI pode ser restringido para pacientes com alto risco.
\end{abstract}

Descritores: Pós-operatório em cirurgia torácica; Redução do tempo de internação.

\section{INTRODUÇÃO}

O desenvolvimento e a aplicação da cirurgia minimamente invasiva possibilita, entre outras vantagens, uma rápida recuperação funcional por parte do paciente e conseqüente encurtamento do tempo de hospitalização. Em cirurgia torácica, e em especial no nosso meio, em função dos custos e prevalência de determinadas doenças, a utilização da vídeo- toracoscopia quase que se restringe a procedimentos periféricos. A sua aplicação na ressecção pulmonar por câncer ainda depende de uma firme avaliação ${ }^{1}$. Por outro lado, existe a percepção da importância de baixar os custos hospitalares face à premente falta de recursos. Isto tem mobilizado a aplicação de protocolos clínicos em pós-operatório com o objetivo de reduzir o tempo de internação, justamente nos países mais ricos. ${ }^{2}$

1. Doutor em Medicina-Pneumologia; Diretor do Serviço de Cirurgia Torácica do Hospital Universitário de Brasília.

2. Cirurgião Torácico do Hospital Universitário de Brasília.

Recebido em 17/07/2002

Aceito para publicação em 29/04/2003

Trabalho realizado no Hospital Universitário de Brasília - DF 
Reduzir o custo da internação hospitalar deveria ser uma preocupação premente no nosso meio, com a vantagem adicional de o menor tempo de internação permitir avançar mais rapidamente a fila de espera nos hospitais públicos com demanda reprimida. O Serviço de Cirurgia Torácica do Hospital Universitário de Brasília iniciou a sua atividade no ano de 1990, e no decurso da década ocorreu importante decréscimo no tempo de internação após cirurgia de ressecção pulmonar, mesmo sem a aplicação de medidas específicas para esse fim. O estudo do levantamento de dados daquele período, e de uma amostragem atual comparada a uma enquête a nível nacional, tem como objetivo quantificar a redução do tempo de internação na nossa Instituição e identificar possíveis fatores relacionados com o restabelecimento mais rápido do paciente em pós-operatório de cirurgia torácica de grande porte.

\section{MÉTODO}

O presente estudo foi realizado no Hospital Universitário de Brasília, que atende exclusivamente um público de baixa renda e capta seus recursos junto ao SUS e Ministério da Saúde. Trata-se de um hospital de porte médio - 300 leitos, cuja demanda por leitos supera em muito a capacidade de atendimento, principalmente nos departamentos de cirurgia e em especial no CTI - seis leitos. Esta indisponibilidade do CTI forçou um equacionamento simples do pósoperatório imediato, desde o início das atividades do Serviço de Cirurgia Torácica. À exceção dos pacientes com alto risco para ressecção pulmonar, seja por co-morbidade seja por função pulmonar limítrofe, a maioria dos pacientes prescinde de CTI e a extubação é levada a cabo na sala de cirurgia. Após o necessário período na sala de recuperação anestésica o paciente é encaminhado para a enfermaria, que conta com enfermagem bem treinada para o atendimento do paciente submetido a procedimentos torácicos, e com monitores e materiais necessários. Os pacientes que recebem analgesia epidural ficam sob estrita vigilância do grupo de anestesiologia. A aplicação de pressão negativa no sistema de drenagem irreversível, no valor 15 a $20 \mathrm{~cm}$ de água, é restrita para os casos submetidos a ressecção pulmonar parcial que apresentam pneumotórax na vigência de fuga aérea. Os critérios para retirada dos drenos pleurais são os usuais, isto é, re-expansão pulmonar completa e ausência de fuga aérea sem preocupação quanto ao débito desde que seja seroso ou sero-hemático. Os pacientes submetidos a pneumonectomia permanecem com um dreno por 24 a 48 horas, que periodicamente é aberto para monitoramento da drenagem e centralização do mediastino. A sondagem vesical de demora não é utilizada de rotina no serviço. $\mathrm{O}$ atendimento fisioterápico é apenas solicitado para casos específicos, não sendo realizado de rotina face às dificuldades logísticas e de demanda.

O estudo foi dividido em duas fases de coleta de dados, ambos retrospectivos e por revisão dos prontuários de pacientes que se submeteram a cirurgia de ressecção compreendendo no mínimo dois segmentos pulmonares ou de tumores do mediastino. Deste estudo estão excluídos os paciente submetidos a cirurgia sobre o esôfago, a cirurgia redutora do volume pulmonar ou procedimentos vídeo-assistidos. A fase I do estudo analisa uma série consecutiva de 169 pacientes, sendo Grupo I aqueles operados no período de junho de 1990 a dezembro de 1995 e Grupo II os que foram operados no período de janeiro de 1996 a maio de 2000. Os parâmetros analisados para comparação entre os grupos foram: idade, sexo, etiologia da doença, via de acesso, tipo de ressecção, índice de morbidade e mortalidade, técnica de analgesia, tempo com dreno e sem dreno durante a internação, tempo de drenagem superior a cinco dias, e tempo de permanência hospitalar após o procedimento cirúrgico (média e mediana). $\mathrm{O}$ índice de morbidade refere-se a qualquer tipo de complicação peri-operatória, incluindo a re-internação hospitalar, e para mortalidade o óbito ocorrido no período de 30 dias após a realização da operação.

A fase II do estudo analisa uma série consecutiva de 20 pacientes (Grupo III) submetidos a cirurgia torácica de grande porte, a partir de março do ano 2002. O levantamento refere-se ao tipo de ressecção, idade média dos pacientes (e variação), via de acesso, técnica de analgesia, tempo de permanência hospitalar após a retirada do dreno, e tempo de internação (média e mediana). Paralelamente, e com o objetivo de captar um perfil atual e "instantâneo" do tempo de internação a nível nacional, foi enviado via internet uma pequena enquête para $21 \mathrm{Ci}$ rurgiões Torácicos com distribuição geográfica pulverizada pelo Brasil. Além da mesma série de vinte pacientes consecutivos e dos dados acima relacionados, foram solicitadas informações adicionais que permitissem tipificar a instituição e rotina pós-operatória do serviço de origem. Demarcou-se o dia limite para 
retorno das informações, e de forma passiva deu-se como encerrada a enquête passados quinze dias daquela data.

As variáveis estudadas foram comparadas entre os grupos I e II através do teste $t$ de Student, e considerou-se haver diferenças estatisticamente significantes quando $\mathrm{p}<0,05$.

\section{RESULTADOS}

Da fase I do estudo, o grupo I ( $\mathrm{n}=81)$ e grupo II $(\mathrm{n}=88)$ foram semelhantes quanto à idade média dos pacientes, distribuição por sexo, etiologia da doença operada, distribuição pelo tipo de ressecção, mortalidade, índice de complicações, tempo de drenagem e drenagem prolongada ( $>5$ dias $)$ - Tabela 1 . $O$ percentual de pacientes submetidos a pneumonectomia foi de $24,7 \%$ no grupo I e de $27,3 \%$ no grupo II. A toracectomia esteve associada à ressecção pulmonar em quatro pacientes do grupo I e em seis pacientes do grupo II.

O tempo médio de internação pós-cirurgia torácica de grande porte foi menor e estatisticamente significante no grupo II $(5,1 \pm 3,0$ dias $)$ comparativamente ao grupo I $(7,6 \pm 3,2$ dias, $\mathrm{p}<0,001)$. Os outros parâmetros estudados em que se observaram diferenças com significância estatística foi o tempo de internação após a retirada do dreno, a técnica de analgesia e o tipo de toracotomia - Tabela 2. Enquanto no grupo I a utilização de bloqueio epidural foi excepcional, no grupo II ocorreu em quase metade dos casos. Quanto ao tipo de toracotomia, e ao longo da década, houve uma transição da toracotomia pósterolateral circum escapular para a toracotomia vertical, passando pela toracotomia lateral.

Da fase II do estudo, o grupo III $(n=20)$ teve como média de idade 48 anos (variação 7 a 83 anos) e ocorreu um óbito - embolia pulmonar pós pneumonectomia direita. Dez pacientes foram submetidos a pneumonectomia, sendo sete direitas e três à esquerda, nove pacientes sofreram lobectomia e um paciente submeteu-se à ressecção de tumor no estreito torácico superior por cervicotoracotomia. Dois pacientes submeteram-se à ressecção pulmonar na sequiência da quimioterapia de indução e em um paciente realizou-se lobectomia em braçadeira. Apenas um paciente realizou o pós-operatório imediato na UTI, por apresentar $\mathrm{VEF}_{1}$ de $750 \mathrm{ml}$ na avaliação funcional para pneumonectomia esquerda por pulmão destruído com infecção de repetição e hemoptise grave, associado a fibro-atelectasia em lobo superior direito. A criança de sete anos de idade teve a lobectomia inferior esquerda por bronquiectasias associada à correção de pronunciado pectus excavatum no mesmo tempo anestésico. Em $90 \%$ das operações a via de acesso foi por toracotomia vertical com preservação muscular. A analgesia epidural foi efetiva em $75 \%$ dos casos. O tempo médio de internação foi de 4,2 dias e a mediana de quatro dias. A alta foi dada no dia da retirada do dreno em oito pacientes (40\%), no dia seguinte em três pacientes, e dois dias após a retirada do dreno em sete pacientes.

Dos 21 cirurgiões solicitados a responder à pequena enquête, oito enviaram as respostas referentes ao tempo de internação dos últimos 20 pacien-

Tabela 1 - Variáveis estudadas que foram semelhantes nos grupos da fase I.

\begin{tabular}{|c|c|c|}
\hline Variáveis & $\begin{array}{c}\text { Grupo I } \\
(\mathbf{n}=\mathbf{8 1})\end{array}$ & $\begin{array}{c}\text { Grupo II } \\
(\mathbf{n}=\mathbf{8 8})\end{array}$ \\
\hline Idade média (anos) & $49,3 \pm 17,3$ & $48,2 \pm 18,0$ \\
\hline $\operatorname{Sexo}(\mathrm{F} / \mathrm{M})$ & 39 / 42 & $42 \overline{/} 46$ \\
\hline Doença neoplásica/supurativa (n) & 46 / 30 & $50 / 32$ \\
\hline Pneumonectomia/lobectomia/piramidectomia (n) & $20 / 37 / 4$ & $24 / 39 / 5$ \\
\hline Ressecção multisegmentar/Tu mediastino (n) & $16 / 4 \quad 17 / 3$ & \\
\hline Mortalidade & $(3,7 \%)$ & $(4,5 \%)$ \\
\hline Índice de complicações & $(13,6 \%)$ & $(18,1 \%)$ \\
\hline Dias com dreno & $3,4 \quad 3,6$ & \\
\hline Drenagem $>5$ dias & 4 & 8 \\
\hline
\end{tabular}

Obs.: Ressecção multisegmentar é anatômica e compreende bilobectomia, lingulectomia associada a lobectomia inferior entre outras. 
Tabela 2 - Variáveis estudadas e índices de significância estatística nos grupos da fase I.

\begin{tabular}{|c|c|c|c|}
\hline Variáveis & $\begin{array}{c}\text { Grupo I } \\
(n=81)\end{array}$ & $\begin{array}{c}\text { Grupo II } \\
(\mathbf{n}=\mathbf{8 8})\end{array}$ & $\mathbf{p}$ \\
\hline Tempo médio de internação (dias) & $7,6 \pm 3,2$ & $5,1 \pm 3,0$ & 0,001 \\
\hline Variação & $3-19$ & $2-17$ & - \\
\hline Mediana & 7 & 4 & - \\
\hline Tempo de internação sem dreno (dias) & $3,8 \pm 2,4$ & $1,6 \pm 1,7$ & 0,001 \\
\hline Bloqueio intercostal/Bloqueio epidural (n) & $79 \overline{/} 2$ & $47 \overline{/} 41$ & 0,001 \\
\hline \multicolumn{4}{|l|}{ Tipo de via de acesso (n) } \\
\hline Toracotomia póstero-lateral & 48 & 5 & 0,001 \\
\hline Toracotomia lateral & 28 & 49 & 0,009 \\
\hline Toracotomia anterior & 3 & 3 & 0,6 \\
\hline Toracotomia vertical & 0 & 26 & 0,001 \\
\hline Esternotomia mediana & 2 & 5 & 0,4 \\
\hline
\end{tabular}

tes submetidos a cirurgia torácica maior e aspectos da rotina do serviço. $O$ tempo médio de internação variou entre cinco e nove dias. Quanto ao tipo de Instituição de origem das séries, dois eram de hospitais universitários, dois da rede pública, três originadas de atividade mista e uma era puramente da prática privada. Apenas dois cirurgiões usaram toracotomia com preservação muscular de forma rotineira, e seis têm a via epidural como preferencial de analgesia. A drenagem pós pneumonectomia foi utilizada na quase totalidade dos casos. Em todos os serviços o pós-operatório imediato foi realizado em UTI ou unidade similar.

\section{DISCUSSÃO}

A redução do tempo de internação médio de 7,6 dias (mediana 7) da primeira metade da década de noventa para 5,1 dias (mediana 4) no secundo período não decorreu em virtude de um plano específico para esse fim. Estes resultados contrastam com os obtidos pelo grupo de Zehr ${ }^{2}$ e de Wright ${ }^{3}$ que obtiveram uma queda na média de internação de oito para 6,4 dias e de 10,6 para 7,5 dias, respectivamente, com a aplicação de medidas que visavam a redução dos custos hospitalares. Embora retrospectivo, portanto menos sensível na captação uniforme de dados relacionados a complicações pós-operatórias, o presente estudo não detectou diferenças de morbidade em especial no que tange à re-internação. Este é um aspecto importante da análise, uma vez que no grupo II com mediana de internação de quatro dias, no mínimo metade dos pacientes obtiveram alta entre o segundo e o quarto dia de pós-operatório. Mas como os critérios de alta foram os mesmos para os grupos, isto é, o completo restabelecimento das condições gerais e cardio-respiratórias, não era de se esperar complicações nos pacientes que obtiveram alta mais precocemente. Duas variáveis emergem do estudo como fatores que colaboraram para a eventualmente mais rápida recuperação funcional dos pacientes: a introdução da analgesia por cateter epidural e mudanças na via de acesso. Na toracotomia póstero-lateral seccionava-se transversalmente o músculo grande dorsal e parcialmente os músculos trapézio e rombóide com liberação parcial da inserção costal do músculo serrátio anterior, e obtinha-se ampla abertura torácica com desarticulação costo-vertebral. Enquanto que na toracotomia lateral a secção atinha-se ao músculo grande dorsal e o afastamento costal não incluía a desarticulação. Com a introdução da toracotomia vertical sem secção ou desinserção muscular, passouse a trabalhar por uma reduzida abertura torácica que apenas permite a introdução da mão cerrada do cirurgião, fazendo-se mais necessária a utilização de luz frontal ou por fibra ótica.

Melhor controle da dor no pós-operatório de cirurgia torácica tem sido proporcionado pelo uso da via epidural para analgesia. ${ }^{4} \mathrm{O}$ cateter é colocado imediatamente antes da toracotomia e usualmente mantido por 48 horas. A utilização de toracotomia com menor afastamento do gradil costal, e com preservação muscular, minimiza o trauma operatório e pode permitir a mobilização precoce do conjunto hemitórax e cintura escapular. ${ }^{5}$ No grupo II da fase I do estudo estes elementos não tiveram aplicação sistemática, 
embora tenham sido os únicos que atingiram significância estatística na comparação entre os grupos. A redução da média de 3,8 dias entre a retirada dos drenos e a alta no grupo I para 1,6 dias no grupo II, pode estar não apenas relacionada ao melhor controle da dor e redução do trauma operatório, resultando melhores condições clínicas para a alta. Pode ter havido alguma outra de influência não mensurável como, por exemplo, a maior experiência e confiança do grupo cirúrgico ao longo da década. Num período contemporâneo ao desenvolvimento das técnicas minimamente invasivas, em que se observa dramática redução no tempo de internação mesmo em cirurgias sobre o coração, certamente houve a percepção que na ressecção pulmonar, mesmo por via standard, sete a 10 dias de permanência hospitalar não precisaria ser a regra.

No grupo III da fase II do estudo, que representa um "instantâneo" recente do tempo de internação após cirurgia torácica de grande porte, agora com utilização sistemática da toracotomia vertical com preservação muscular e uso mais efetivo da analgesia epidural, além da conscientização de que paciente restabelecido pode ter alta a qualquer tempo, obteve-se nova redução no tempo médio de internação - 4,2 dias. Neste grupo de 20 pacientes com $50 \%$ pneumonectomizados, estes mais propensos a morbidade peri-operatória, 17 pacientes $(85 \%)$ obtiveram alta até ao $5^{\circ}$ dia de pós-operatório. A análise do conjunto de dados da enquête entre os vários cirurgiões torácicos mostra uma média de internação mais elevada, no entanto dentro de um padrão usual, e que o uso da analgesia epidural é quase generalizado. Contrasta com a nossa série, o uso pouco difundido da toracotomia com preservação muscular, e um outro elemento de relevância da rotina de pós-operatório imediato - o envio obrigatório do paciente para a UTI ou similar. O uso seletivo da UTI foi um aspecto destacado como medida fundamental para o sucesso do protocolo para redução do tempo de internação implementado por Cerfolio e colaboradores. ${ }^{6}$ Este grupo estabeleceu um protocolo que objetivava a alta em torno do $4^{\circ}$ ou $5^{\circ}$ dia de pós-operatório de ressecções pulmonares, em que o paciente não apenas estava ao par dessa meta como a família foi instruída sobre as ações importantes, tal como receber o paciente no quarto oriundo diretamente do centro cirúrgico. A maioria dos pacientes prescindiu da UTI e, apesar da via de acesso rotineira tenha sido a toracotomia póstero-lateral sem preservação do gran- de dorsal, a média do tempo de internação foi de quatro dias $-65 \%$ dos pacientes obtiveram alta no $4^{\circ}$ dia ou mais cedo. O retorno direto do paciente para o quarto ou a enfermaria permite a deambulação precoce, a maior liberdade de se alimentar no mesmo dia da cirurgia, e no dia seguinte já ser capaz de realizar a higiene pessoal. No entanto, o paciente que não necessita de cuidados intensivos e mesmo assim vai para a UTI, pode permanecer desnecessariamente intubado por longo período, não terá liberdade de sair do leito mesmo que extubado, o ambiente pouco acolhedor não lhe dará nenhum apetite para se alimentar, e no dia seguinte o banho será no leito. Por outro lado, não utilizar obrigatoriamente a UTI não apenas reduz os custos operacionais diretos e indiretos da internação como pode proporcionar uma percepção da real magnitude de uma ressecção pulmonar por parte do paciente, que afinal percebe não o incapacitar momentaneamente e que logo poderá ir para casa.

Ao lado do controle da dor, do uso de vias de acesso menos traumáticas, e de um plano anestésico para extubação na sala de cirurgia com obtenção de um acordar que faculte a efetiva eliminação de secreções, é fundamental a implementação de estratégias e facilidades para deambulação precoce e agressiva, com vistas ao rápido restabelecimento do paciente. ${ }^{7}$ Entretanto, o paciente pode estar completamente recuperado e em condições clínicas de alta, mas a necessidade da manutenção da drenagem pela presença de escape aéreo torna-se um óbice à liberação da internação. Apesar de cirurgia tecnicamente meticulosa, as fístulas bronquíolo-pleurais que demandam drenagem prolongada ocorrem com certa freqüência, em especial no grupo de pacientes operados por câncer de pulmão portadores de enfisema pulmonar. Existem evidências claras de que a drenagem sub-aquática passiva, e na condição do pulmão remanescente apresentar-se completamente expandido, é mais efetiva no fechamento daquelas fístulas em contra ponto ao uso imediato da pressão negativa no sistema. ${ }^{8}$ Medidas intra-operatórias para adequar o volume do espaço pleural ao tamanho do pulmão remanescente, como a confecção de tenda pleural apical no caso de lobectomia superior ou a realização de pneumoperitônio no caso de bilobectomia ou lobectomia inferior, são bastante efetivas na profilaxia de escapes aéreos prolongados e permitem reduzir o tempo de permanência dos drenos e da hospitalização. ${ }^{9,10}$ Para os pacientes com fuga aé- 
rea persistente, e que por este motivo são mantidos hospitalizados, existe a alternativa de seguimento ambulatorial após a instalação da válvula de Heimlich.

Questões relativas ao manejo do paciente pneumonectomizado sugerem persistente influência do treinamento de origem sobre cirurgião torácico. A grande maioria dos cirurgiões torácicos respondeu que drena de rotina o hemitórax desabitado, nós próprios o fazemos, e poucos questionamos esse hábito como talvez não questionemos uma permanência de quatro ou cinco dias quando tudo está muito bem a partir do segundo dia pós-operatório. Do grupo III todos os 10 casos de pneumonectomia foram drenados de acordo com a rotina do serviço, e pese que uma boa parte dos casos tenha tido liberação pulmonar pelo plano extra-pleural, em nenhum foi necessário tomar medidas terapêuticas em função da drenagem monitorada. Como a centralização do mediastino pode ser obtida através medidas mais simples ao final do procedimento operatório, e da literatura é sabido dos bons resultados com ou sem drenagem pós pneumonectomia, a opção por não drenar pode tornar-se atrativa por ser menos um óbice à deambulação precoce do paciente. $\mathrm{O}$ tempo de permanência nós questionamos, e por isso temos dado alta no segundo ou terceiro dia de pós-operatório se o paciente se apresentar funcionalmente recuperado, sem temer hipotéticas complicações como o edema pulmonar pós-pneumonectomia.

Tovar $^{11}$ não apenas impressiona, como deixa claro que a redução do tempo de hospitalização não foi o objetivo de um programa de recuperação acelerada, mas antes uma medida da rápida capacidade da restauração funcional. Numa série consecutiva de 65 pacientes submetidos a ressecção pulmonar - 45 lobectomias, 11 pneumonectomias e oito bilobectomias, obteve um tempo médio de internação de 1,2 dias em que 54 pacientes tiveram alta no dia seguinte da operação e dois foram para casa no mesmo dia. Desta série, 30 pacientes tinham mais de 70 anos de idade. Esse programa incluía um firme processo de esclarecimento ao paciente e família, via de acesso por minitoracotomia com preservação muscular, ressecção pulmonar assistida por vídeo, técnicas associadas de analgesia, extubação na sala de cirurgia, e deambulação no mesmo dia da operação. A utilização de técnica minimamente invasiva já encontra razoável respaldo na literatura no que tange à ressecção pulmonar por câncer no estádio IA, não apenas por ser válida do ponto de vista oncológico, mas também pelo menor trauma e repercussão sobre o sistema imunológico. ${ }^{12}$ Minimizar a depressão do sistema imunológico pode ser a chave para não perder o controle das eventuais micrometástases que impedem a sobrevida de 5 anos em $25 \%$ dos pacientes operados naquele estádio. ${ }^{13}$ No nosso meio, não são poucos os obstáculos impostos pela deficiência de recursos no sistema público e pela retração no sistema privado ao uso dos insumos necessários à cirurgia assistida por vídeo. No entanto, parece inexorável que esse é o caminho a ser seguido, com saldo certamente positivo até do ponto de vista econômico.

Em conclusão, este estudo embora retrospectivo e de diferentes períodos, sugere que a maioria dos pacientes submetidos a cirurgia torácica de grande porte pode evoluir com rápido restabelecimento funcional, e obter alta em torno do $3^{\circ}$ ou $4^{\circ}$ dia de pós-operatório, quando medidas como otimização do controle da dor e minimização do trauma operatório são adotadas. Também a criteriosa extubação na sala de cirurgia com dispensa da UTI e deambulação precoce podem ter papel relevante na recuperação acelerada do paciente. Existe espaço para desenvolvimento de programas específicos com implementação de medidas adicionais que promovam a recuperação ainda mais rápida das condições gerais do paciente.

\section{AGRADECIMENTOS}

Aos cirurgiões torácicos pela pronta colaboração na enquête: Artur Gomes Neto (AL), Carlos Alberto Almeida de Araújo (RN), José Antônio de Figueiredo Pinto (RS), José Ribas Milanez de Campos (SP), Manoel Ximenes Netto (DF), Mauro Natel de Oliveira (MS), Pedro Luís Reis Crotti (MT) e Rui Haddad (RJ). 


\begin{abstract}
Background: There is a direct relation between hospital costs and hospital length of stay after the operation. On the other hand, reduced stay increases productivity of public hospitals with high service demanding. Our objective is to identify factors determining the decrease in hospital stay after major thoracic surgery. Methods: A two-phase retrospective study was conducted on analysis of medical records. In the first phase, data on length of hospital stay and related factors were collected from a consecutive series of 169 patients divided into group I $(n=81)$ - patients operated on between June 1990 and December 1995, and group II $(n=88)$-patients operated on from January 1996 through May 2000. In the second phase, data were collected from a consecutive series of 20 patients (group III) starting backwards from March 2002, for analysis and comparison with an Internet survey sent to 21 thoracic surgeons. Results: Intensive care unit was unecessary for most patients in immediate post operative period. The mean hospital stay decreased from 7.6 days (median 7$)$ in group I to 5.1 days (median 4) in group II ( $p<0.001)$. The more frequent utilization of epidural analgesia and less traumatic thoracothomy in group II reached statistic significance ( $p<0.001)$. In group III, the mean hospital stay was 4.2 days (median 4), and there was a more efective use of epidural analgesia (75\%) and muscle-sparing thoracothomy (90\%). Eight thoracic surgeons answered the survey: the mean hospital stay varied from five to nine days and all patients were sent to intensive care or similar units. Only two surgeons utilize muscle-sparing thoracothomy. Conclusions: This study confirms that pain control and less traumatic surgical approach are important for faster functional recovery of patients. It suggests that the IC units may be used only for selected patients.
\end{abstract}

Key words: Post operative period in thoracic surgery; Early hospital discharge; Early discharge following major thoracic surgery; Identification of related factors.

\section{REFERÊNCIAS}

1. Landreneau RJ, Mack MJ, Dowling RD, Luketich JD, Keenan RJ, Ferson PF et al. The role of thoracoscopy in lung cancer management. Chest 1998;113:6S-12S.

2. Zehr KJ, Dowson PB, Yang SC, Heitmiller RF. Standardized clinical care pathways for major thoracic cases reduce hospital costs. Ann Thorac Surg 1998;66:914-9.

3. Wright CD, Wain JC, Grillo HC, Moncure AC, Macaluso SM, Mathisen DJ. Pulmonary lobectomy patient care pathway: a model to control cost and maintain quality. Ann Thorac Surg 1997;64:299-302.

4. Peeters-Asdourian C, Cruptas S. Choices in pain management following thoracotomy. Chest 1999;115(5S):122S-124S.

5. Benedetti F, Vighetti S, Ricco C, Amanzio M, Bergamasco L, Casadio C et al. Neurophysiologic assessment of nerve impairment in posterolateral and muscle-sparing thoracotomy. $\mathbf{J}$ Thorac Cardiovasc Surg 1998;115(4):841-7.

6. Cerfolio RJ, Pickens A, Bass C, Katholi C. Fast-tracking pulmonary resections. J Thorac Cardiovasc Surg 2001;122:318-24.

7. Engelman RM. Mechanisms to reduce hospital stays. Ann Thorac Surg 1996;61:S26-S29.

8. Cerfolio RJ, Bass C, Katholi CR. Prospective randomized trial compares suction versus water seal for air leaks. Ann Thorac Surg 2001;71:1613-7.
9. Robinson LA, Preksto D. Pleural tenting during upper lobectomy decreases chest tube time and total hospitaization days. J Thorac Cardiovasc Surg 1998;115:319-27.

10. Cerfolio RJ, Holman WL, Katholi CR. Pneumoperitoneum after concomitant resection of the right middle and lower lobes (bilobectomy). Ann Thorac Surg 2000;70:942-7.

11. Tovar EA. One-day admission for major lung resections in septuagenarians and octogenarians: a comparative study with a younger cohort. Eur J Cardiothorac Surg 2001;20(3):449-53.

12. Yim APC, Wan S, Lee TW, Arifi AA. VATS lobectomy reduces cytokine responses compared with conventional surgery. Ann Thorac Surg 2000;70:243-7.

13. Kaseda S, Aoki T, Hangai n, Shimizu K. Better pulmonary function and prognosis with vídeo-assisted thoracic surgery than with thoracotomy. Ann Thorac Surg 2000;70:1644-6.

Endereço para correspondência:

Nuno F. Ferreira de Lima, SHIS QI 15 Conj.11 Cs13

CEP 71635-310 Brasília DF

Tels. (61) 3643129-3641587-2453445

Fax: (61) 3474940

E-mail:nunolima@globo.com 